K. Diederich and E. Mazzilli

Nagoya Math. J.

Vol. 163 (2001), 215-227

\title{
ZERO VARIETIES FOR THE NEVANLINNA CLASS ON ALL CONVEX DOMAINS OF FINITE TYPE
}

\author{
KLAS DIEDERICH AND EMMANUEL MAZZILLI
}

\begin{abstract}
It is shown, that the so-called Blaschke condition characterizes in any bounded smooth convex domain of finite type exactly the divisors which are zero sets of functions of the Nevanlinna class on the domain. The main tool is a non-isotropic $L^{1}$ estimate for solutions of the Cauchy-Riemann equations on such domains, which are obtained by estimating suitable kernels of BerndtssonAndersson type.
\end{abstract}

\section{$\S 1$. Introduction and results}

In their article [2] J. Bruna, Ph. Charpentier and Y. Dupain did a large step towards a complete characterization of the zero sets of the Nevanlinna class on all convex domains of finite type in $\mathbb{C}^{n}$. The purpose of this article is to complete their result.

For the convenience of the reader we recall at first briefly the following definitions and facts:

If $D \subset \subset \mathbb{C}^{n}$ is a $\mathcal{C}^{\infty}$ - smoothly bounded domain and $\rho$ a smooth defining function for $D$, then the Nevanlinna class $N(D)$ is

$$
N(D)=\left\{f \in \mathcal{O}(D): \sup _{\varepsilon} \int_{\partial D_{\varepsilon}}|\log | f(z)|| d \sigma_{\varepsilon}(z)<+\infty\right\}
$$

Here $\partial D_{\varepsilon}=\{z: \rho(z)=-\varepsilon\}$ for $\varepsilon>0$ small enough and $d \sigma_{\varepsilon}(z)$ denotes the Euclidean surface measure on $\partial D_{\varepsilon}$. Furthermore, if $X \subset D$ is a complex analytic hypersurface with irreducible decomposition

$$
X=\bigcup_{k} X_{k}
$$

Received May 12, 2000.

2000 Mathematics Subject Classification: 32A35; 32A26; 32A60; 32F32; 32F45; $32 \mathrm{~W} 05$. 
and $n_{k} \in \mathbb{N}$ are positive integers for each $k$, the divisor $\hat{X}:=\left\{X_{k}, n_{k}\right\}$ is said to satisfy the Blaschke condition if

$$
\sum_{k} n_{k} \int_{X_{k}}|\rho(z)| d \mu_{X_{k}}(z)<\infty .
$$

It is a well-known fact, that for any function $f \in N(D)$ the zero divisor $X_{f}$ of $f$ satisfies the Blaschke condition (1.1). And it is conjectured, that the inverse also is always true.

For a survey of the partial positive answers to this conjecture known sofar see [2]. The basic work for $n>1$ was done independently by G. Henkin in [7] and H. Skoda in [9] for the case of strictly pseudoconvex domains in $\mathbb{C}^{n}$.

In [2] the authors consider the class of bounded convex smooth domains $D \subset \subset \mathbb{C}^{n}$ of finite type with the goal of showing, that condition (1.1) characterizes precisely the zero divisors of the functions in $N(D)$. A large part of their work goes, in fact, through for all such domains. However, in order to accomplish the final analytically most critical step of their method of proof (which, actually, is the complete analogue of the method introduced by H. Skoda), they need the following additional condition on the domains $D$ :

Definition 1.1. A smooth convex domain $D \subset \subset \mathbb{C}^{n}$ is called of strict type if there is a constant $c=c(D)$ such that for all $\zeta \in \partial D$ and all $v \in T_{\zeta}^{10} \partial D$ with $|v|=1$ and for all small $t$

$$
c^{-1} \rho(\zeta+t v) \leq \rho(\zeta+t i v) \leq c \rho(\zeta+t v)
$$

If $D$ is in addition of finite type $\leq m$, it is called of finite strict type $\leq m$.

Altogether, J. Bruna, Ph. Charpentier and Y. Dupain showed in [2], that any divisor $\hat{X}$ on a bounded smooth convex domain $D$ of finite strict type in $\mathbb{C}^{n}$ satisfying the Blaschke condition comes from a function $f \in$ $N(D)$. The crucial analytic step, where the strict type condition is needed in [2] is the construction of $\bar{\partial}$ solutions with $L^{1}$ estimates on the boundary (see Theorem 1.3 below).

The analysis on domains $D$ of finite strict type is much easier than on other convex domains of finite type, since for such $D$ the family $T_{\zeta}^{10} \partial D=$ 
$\left\{w \in \mathbb{C}^{n}:\langle\partial \rho(\zeta), w-\zeta\rangle=0\right\}$ for $\zeta \in \partial D$ is a perfect family of holomorphic support functions which can be used in the construction of $\bar{\partial}$-solving integral kernels. The difference between smooth convex domains which are of finite strict type and others which are just of finite type becomes clear, if one compares among the standard model domains the so-called complex pseudoellipsoids

$$
D:=\left\{z: \sum_{k=1}^{n}\left|z_{k}\right|^{2 m_{k}}<1\right\}
$$

with positive integers $m_{k}$ one of which being $>1$, with the real ellipsoids

$$
\hat{D}:=\left\{z: \sum_{k=1}^{n}\left(x_{k}^{2 m_{k}}+y_{k}^{2 q_{k}}\right)<1\right\}
$$

where, of course, $z_{k}=x_{k}+i y_{k}, m_{k}, q_{k}$ are again positive integers, at least one $m_{k}>1$ and the corresponding $q_{k}>m_{k}$. The domains $D$ are of finite strict type, whereas $\hat{D}$ are only of finite type, but not of strict type. Concerning the question, how to do analysis on convex domains of finite type which are not of strict type, $\bar{\partial}$-solving integral kernels for real pseudoellipsoids were constructed and estimated in [6]. In continuation of this work, smooth families of holomorphic support functions with best possible non-isotropic estimates were constructed for all convex domains of finite type in [5]. In [4] they were used for constructing $\bar{\partial}$-solving integral kernels with best possible Hölder estimates. Here we will now use them in order to show essentially by Skoda's method the following

THEOREM 1.2. Let $D \subset \subset \mathbb{C}^{n}$ be a smooth convex domain of finite type. Then there is a constant $C>0$, such that there is for any smooth $(0,1)$-form $\omega$ on $\bar{D}$ with $\bar{\partial} \omega=0$ a smooth function $u$ on $\bar{D}$ such that $\bar{\partial} u=\omega$ and

$$
\int_{\partial D}|u(z)| d \sigma(z) \leq C \int_{D}\|\omega(z)\|_{k} d V(z) .
$$

Here the unisotropic norm $\|\cdot\|_{k}$ for $(0,1)$-forms is defined by

$$
\|\omega(z)\|_{k}=\sup \left\{\frac{|\omega(z)(v)|}{k(z, v)}: v \in \mathbb{C}^{n} \backslash\{0\}\right\}
$$

where $k(z, v)$ is the usual weighted boundary distance of $z$ in $D$ in the direction $v$ (for details see for instance [2]). 
It was already shown in [2], that the following theorem is a consequence of this:

TheOREM 1.3. Let $D$ be as in Theorem 1.2. Then there is for every divisors $\hat{X}=\left\{X_{k}, n_{k}\right\}$ in $D$ satisfying the Blaschke condition (1.1) a function $f \in N(D)$, such that $\hat{X}$ is the zero divisor of $f$.

Remark 1.4. a) The article [3] of Anne Cumenge also contains this theorem. She uses a different approach.

b) This article appeared as a preprint on November 26, 1998, and was distributed as such among many colleagues.

Large parts of the following sections containing the proof of Theorem 1.2 are based on the work done in [2]. However, our situation is more complicated, such that at some stages a different approach has to be used. We will almost always use the same notations as in [2].

\section{$\S 2$. Integral kernels revisited}

For proving Theorem 1.2 we want to use an integral kernel of the type H. Skoda. In order to construct it we use the methods of [1]. For them a $\operatorname{good} \mathcal{C}^{\infty}$-family $S(z, \zeta)$ of support functions on $D$, holomorphic in $z \in \bar{D}$ and $\mathcal{C}^{\infty}$ in $\zeta$ chosen in a suitable neighborhood of $\partial D$ together with the corresponding Leray section $s(z, \zeta)$ is needed. We obtain it from the family $S(z, \zeta)$ constructed in [5]. The necessary estimates for $S$ and $s$ are contained in $[4]$.

More precisely, Proposition 4.1 from [4] says:

Proposition 2.1. Let $D \subset \subset \mathbb{C}^{n}$ be a smooth convex domain of finite type $m$ and $\rho$ a convex defining function of $D$ in a neighborhood $U$ of $\partial D$. Then the function $S(z, \zeta) \in \mathcal{C}^{\infty}(\bar{D} \times U)$, holomorphic in $z$, constructed in [5], has the following property:

Let, for $\zeta \in U, n_{\zeta}$ denote the outer unit normal to the level set $\{\rho=$ $\rho(\zeta)\}$ and let $v$ be any unit vector complex tangential to this level set at $\zeta$. Define

$$
a_{\alpha \beta}(\zeta, v):=\frac{\partial^{\alpha+\beta}}{\partial \lambda^{\alpha} \partial \bar{\lambda}^{\beta}} \rho(\zeta+\lambda v)_{\mid \lambda=0} .
$$


Then there are constants $K, c, d>0$, such that one has for all points $z$ written as $z=\zeta+\mu n_{\zeta}+\lambda v$ with $\mu, \lambda \in \mathbb{C}$ the estimate

(2.1) $\quad \operatorname{Re} S(z, \zeta) \leq-\left|\frac{\operatorname{Re} \mu}{2}\right|-\frac{K}{2}(\operatorname{Im} \mu)^{2}$

$$
-c \sum_{j=2}^{m} \sum_{\alpha+\beta=j}\left|a_{\alpha \beta}(\zeta, v) \| \lambda\right|^{j}+d \sup \{0, \rho(z)-\rho(\zeta)\} .
$$

Remark 2.2. In order to avoid that the constant $d$ appears again and again in the estimates of this article, we replace from now on the original $S(z, \zeta)$ by $\frac{1}{2 d} S(z, \zeta)$ calling this new function again $S(z, \zeta)$.

In (5) and (6) of [4] $\mathcal{C}^{\infty}$ functions $Q_{j}(z, \zeta), j=1, \ldots, n$, holomorphic in $z$, were defined, such that

$$
S(z, \zeta)=\sum_{j=1}^{n} Q_{j}(z, \zeta)\left(z_{j}-\zeta_{j}\right)
$$

for $(z, \zeta) \in \bar{D} \times U$.

Let us now choose a number $\eta_{0}>0$ such that $U_{0}:=\{w:|\rho(w)|<$ $\left.\eta_{0}\right\} \subset U$ and a $\mathcal{C}^{\infty}$ cut-off function $\chi$ with $\chi(w)=0$ for $\rho(w) \geq-\frac{\eta_{0}}{2}$ and $\chi(w)=1$ for $\rho(w) \leq-\eta_{0}$. We put for $(\zeta, z) \in \bar{D} \times \bar{D}$

$$
s(\zeta, z):=-\rho(z) \sum_{i=1}^{n}\left(\bar{\zeta}_{i}-\bar{z}_{i}\right) d \zeta_{i}+(1-\chi(z)) \overline{S(\zeta, z)} \sum_{i=1}^{n} Q_{i}(\zeta, z) d \zeta_{i}
$$

and

$$
\mathcal{Q}(\zeta, z):=\frac{1}{\rho(\zeta)}\left[(1-\chi(\zeta)) \sum_{i=1}^{n} Q_{i}(z, \zeta) d \zeta_{i}+\chi(\zeta) \sum_{i=1}^{n} \frac{\partial \rho}{\partial \zeta_{i}}(\zeta) d \zeta_{i}\right]
$$

For any compact subset $A \subset D$ we obviously can find a constant $K>0$ such that

$$
|\langle s(\zeta, z), \zeta-z\rangle| \geq K\|\zeta-z\|^{2}
$$

for all $\zeta \in \bar{D}$ and $z \in A$. Furthermore, $s(\zeta, z)$ is holomorphic in $z$ for $\zeta \in D$. 
Following the method of Berndtsson-Andersson [1], we define the kernel

$$
\begin{aligned}
& \quad K(\zeta, z):= \\
& c_{n} \sum_{k=0}^{n-1} c_{k} \frac{s \wedge\left(\bar{\partial}_{\zeta} s\right)^{n-1-k} \wedge\left(\bar{\partial}_{\zeta} \mathcal{Q}\right)^{k}}{\langle s(\zeta, z), \zeta-z\rangle^{n-k}} \\
& \quad \times \frac{(\rho(\zeta))^{k+1}}{\left[\rho(\zeta)+(1-\chi(\zeta)) S(z, \zeta)+\chi(\zeta) \sum_{i=1}^{n} \frac{\partial_{\rho}}{\partial_{\zeta_{i}}}(\zeta)\left(z_{i}-\zeta_{i}\right)\right]^{k+1}}
\end{aligned}
$$

Observe, that because of Proposition 2.1 and the classical estimate for the last sum in the denominator, the expression $[\cdots]$ in the denominator does not have zeros for $\zeta \in \bar{D}$ and $z \in D$. Therefore, and because of $(2.5)$, the theorem of Berndtsson-Andersson says

Proposition 2.3. Let $\omega$ be a $\bar{\partial}$-closed $(0,1)$-form smooth on $\bar{D}$, and put

$$
u(z):=\int_{D} \omega(\zeta) K(\zeta, z)
$$

for $z \in D$. Then $\bar{\partial} u=\omega$ on $D$.

\section{$\S 3$. Estimation of the solution to the $\bar{\partial}$-equation}

We would like to know at first, that for $\omega$ as in Proposition 2.3 the function $u$ from (2.7) is continuous up to $\partial D$ and its boundary values are given by the formula

$$
u(z)=c_{n} \int_{D} \omega(\zeta) K(\zeta, z) .
$$

According to the theorem of Skoda it suffices to prove for this that $K(\zeta, z)$ is uniformly integrable with respect to $\zeta$ for $z \in \partial D$.

However, for these $z$ we have

$$
s(\zeta, z)=\overline{S(\zeta, z)} \sum_{i=1}^{n} Q_{i}(\zeta, z) d \zeta_{i}
$$

and $Q_{i}(\zeta, z)$ is holomorphic in $\zeta$. Hence

$$
s \wedge \bar{\partial}_{\zeta} s=0
$$


such that it suffices to use $k=n-1$ in the definition of $K(\zeta, z)$ and we obtain for $z \in \partial D$

$$
\begin{aligned}
& K(\zeta, z)= \\
& c_{n} \frac{\sum_{i=1}^{n} Q_{i}(\zeta, z) d \zeta_{i} \wedge\left(\bar{\partial}_{\zeta} \mathcal{Q}\right)^{n-1}[\rho(\zeta)]^{n}}{S(\zeta, z)\left[\rho(\zeta)+(1-\chi(\zeta)) S(z, \zeta)+\chi(\zeta) \sum_{i=1}^{n} \frac{\partial_{\rho}}{\partial_{\zeta_{i}}}(\zeta)\left(z_{i}-\zeta_{i}\right)\right]^{n}}
\end{aligned}
$$

Observe, that for $z \in \partial D$ according to Proposition 2.3 the denominator becomes 0 only for $\zeta=z$ (if we would have used the classical support function as used for instance in [2], then there could be whole pieces of real lines in $\partial D$ as zeros of the denominator in the case of convex domains $D$ of finite type which are not of strict type). Therefore, the corresponding arguments of Section 4 of Bruna-Charpentier-Dupain apply. Hence, for showing the desired uniform integrability of $K(\zeta, z)$, it suffices according to (24) of [2] to prove

LEMMA 3.1. If $m$ denotes the type of $D$, then one for $\varepsilon>0$ sufficiently small

$$
\int_{D \cap P_{\varepsilon}(z)}|K(\zeta, z)| d V(\zeta)=O\left(\varepsilon^{1 / m}\right)
$$

for all $z \in \partial D$. Here $P_{\varepsilon}(z)$ denotes the pseudoball at $z$ with radius $\varepsilon$.

We will reduce the proof of this lemma to two other lemmas. We introduce the notation

$$
\begin{aligned}
Q(\zeta, z) & :=\sum_{i=1}^{n} Q_{i}(\zeta, z) d \zeta_{i} \\
Q(z, \zeta) & :=\sum_{i=1}^{n} Q_{i}(z, \zeta) d \zeta_{i}
\end{aligned}
$$

Notice, that for $\varepsilon>0$ small enough and $z \in \partial D$ one has $P_{\varepsilon}(z) \subset U_{0}$. Hence, $\chi(\zeta)=0$ for $\zeta \in P_{\varepsilon}(z)$ and the kernel $K$ can be simplified in the following way:

with

$$
K(\zeta, z)=K_{1}(\zeta, z)+K_{2}(\zeta, z)
$$


and

$$
K_{2}(\zeta, z)=\frac{Q(\zeta, z) \wedge\left(\bar{\partial}_{\zeta} Q(z, \zeta)\right)^{n-1} \rho(\zeta)}{S(\zeta, z)[\rho(\zeta)+S(z, \zeta)]^{n}}=: \frac{W_{2}(\zeta, z)}{S(\zeta, z)[\rho(\zeta)+S(z, \zeta)]^{n}}
$$

We want to show at first the following estimate small)

LEMMA 3.2. We have uniformly in $z \in \partial D$ and in $\varepsilon>0$ (sufficiently

$$
\int_{D \cap P_{\varepsilon}(z)}\left|W_{i}(\zeta, z)\right|=O\left(\varepsilon^{1 / m+n+1}\right)
$$

for $i=1,2$.

Proof. We treat only the case $i=1$, since the other case is similar. We have

$$
W_{1}(\zeta, z)=Q(\zeta, z) \wedge Q(z, \zeta) \wedge \bar{\partial} \rho(\zeta) \wedge\left[\bar{\partial}_{\zeta} Q(z, \zeta)\right]^{n-2}
$$

We choose $\varepsilon$-extremal coordinates $w=\left(w_{1}, \ldots, w_{n}\right)$ at $z$ and transform the forms $Q(z, \zeta)$ and $Q(z, \zeta)$ by pulling back the $\zeta$-variable, obtaining

$$
Q^{*}(w, z):=\sum_{i=1}^{n} Q_{i}(\zeta(w), z) d \zeta_{i}(w), Q^{*}(z, w):=\sum_{i=1}^{n} Q_{i}(z, \zeta(w)) d \zeta_{i}(w) .
$$

We write

$$
Q^{*}(w, z)=\sum_{i=1}^{n} Q_{i}^{*}(w, z) d w_{i}, Q^{*}(z, w)=\sum_{i=1}^{n} Q_{i}^{*}(z, w) d w_{i} .
$$

We then can estimate $\left|W_{1}(\zeta, z)\right|$ from above by the sum over all terms of the form

$$
\left|Q_{i_{1}}^{*}(w, z)\right|\left|Q_{i_{2}}^{*}(z, w)\right|\left|\frac{\partial \rho}{\partial \bar{w}_{j_{2}}}\right| \prod_{l=3}^{n}\left|\frac{\partial Q_{i_{l}}^{*}(z, w)}{\partial \bar{w}_{j_{l}}}\right|
$$

where $\left(i_{1}, \ldots, i_{n}\right)$ is a multiindex with values from $\{1, \ldots, n\}$ and $i_{l} \neq i_{k}$ for $k \neq l$ and $\left(j_{2}, \ldots, j_{n}\right)$ is a multiindex with values in $\{1, \ldots, n\}$ with $j_{k} \neq j_{l}$ for $k \neq l$.

Each single term in (3.6) can be estimated as in [4] using Proposition 3.1 from [4], which consists of material mostly collected from results of McNeal from [8]. Namely, according to Proposition 3.1, iv) and vii) of [4] we have

$$
\left|\frac{\partial \rho}{\partial \bar{w}_{j_{2}}}(w)\right| \lesssim \frac{\varepsilon}{\tau_{j_{2}}(z, \varepsilon)} \forall \zeta(w) \in P_{\varepsilon}(z)
$$


and Lemma 5.1 of [4] gives

$$
\left|\frac{\partial Q_{i_{l}}^{*}}{\partial \bar{w}_{j_{l}}}(z, w)\right| \lesssim \frac{\varepsilon}{\tau_{i_{l}}(z, \varepsilon) \tau_{j_{l}}(z, \varepsilon)} \forall \zeta(w) \in P_{\varepsilon}(z) .
$$

Furthermore, the same lemma also contains

$$
\left|Q_{i_{l}}^{*}(z, w)\right| \lesssim \frac{\varepsilon}{\tau_{i_{l}}(z, \varepsilon)} \forall \zeta(w) \in P_{\varepsilon}(z) .
$$

It remains to estimate the first factor in (3.6). This is a simplified version of Lemma 5.4 of [4]. We have

$$
\left|Q_{k}^{*}(w, z)\right| \lesssim \frac{\varepsilon}{\tau_{k}(z, \varepsilon)}
$$

where the $\tau_{k}$ are always the weighted directional radii of the pseudoballs also used in [2].

Altogether we get for each expression (3.6) the upper estimate by

$$
\frac{\varepsilon}{\tau_{i_{1}}(z, \varepsilon)} \frac{\varepsilon}{\tau_{i_{2}}(z, \varepsilon)} \frac{\varepsilon}{\tau_{j_{2}}(z, \varepsilon)} \prod_{l=3}^{n} \frac{\varepsilon}{\tau_{i_{l}}(z, \varepsilon) \tau_{j_{l}}(z, \varepsilon)} .
$$

If we put this together into $W_{1}(\zeta, z)$ we get

$$
\left|W_{1}(\zeta, z)\right| \lesssim \varepsilon^{n+1} \tau_{n}(z, \varepsilon) \prod_{l=1}^{n} \frac{1}{\tau_{l}^{2}(z, \varepsilon)} .
$$

The estimate of the lemma for $i=1$ follows directly from this.

In order to formulate the next lemma, we choose a constant $C_{1}>0$ such that $C_{1} P_{\varepsilon / 2}(z) \supset \frac{1}{2} P_{\varepsilon}(z)$ for all $z, \varepsilon$ (see Proposition 3.1,ii) of [4]) and put for positive integers $i$

$$
P_{\varepsilon}^{i}(z):=C_{1} P_{2^{-i} \varepsilon}(z) \backslash P_{2^{-i} \varepsilon}(z) .
$$

Then the family $\left\{P_{\varepsilon}^{i}(z)\right\}$ is a covering of $P_{\varepsilon}(z)$, see (11) of [4].

Next we want to show

LEMMA 3.3. One has

i) $|S(\zeta, z)| \gtrsim 2^{-i} \varepsilon$

ii) $|\rho(\zeta)+S(z, \zeta)| \gtrsim 2^{-i} \varepsilon$

uniformly in $z \in \partial D, \zeta \in P_{\varepsilon}^{i}(z) \cap D$ and all $i$. 
Proof. For showing i) we write $\zeta=\mu n_{z}+\lambda v$ as in Proposition 2.1. According to Proposition 3.1 iii) there is a uniform constant $k_{1}$ such that in either of the following cases

(1) $|\mu| \leq k_{1} \tau\left(z, n_{z}, 2^{-i} \varepsilon\right)$,

(2) $|\lambda| \leq k_{1} \tau\left(z, v, 2^{-i} \varepsilon\right)$

necessarily $\zeta \in \frac{1}{2} P_{2^{-i} \varepsilon}(z)$. In other words, for $\zeta \notin \frac{1}{2} P_{2^{-i} \varepsilon}(z)$, one has

$$
|\lambda| \geq k_{1} \tau\left(z, v, 2^{-i} \varepsilon\right) \text { or }|\mu| \geq k_{1} \tau\left(z, n_{z}, 2^{-i} \varepsilon\right) .
$$

We now show at first that for any $k_{0}<k_{1}$ the inequality $|\lambda| \geq k_{0} \tau\left(z, v, 2^{-i} \varepsilon\right)$ implies i). Namely, we immediately get from Proposition 2.1 together with Proposition $3.1 \mathrm{vi}$ ) of [4] the estimate

$$
|S(\zeta, z)| \geq c k_{0}^{m} \sum_{j=2}^{m} \sum_{\alpha+\beta=j}\left|a_{\alpha \beta}(z, v)\right| \tau^{j}\left(z, v, 2^{-i} \varepsilon\right) \gtrsim 2^{-i} \varepsilon .
$$

If, on the other hand, $|\lambda| \leq k_{0} \tau\left(z, v, 2^{-i} \varepsilon\right)$, we must have $|\mu| \geq k_{1} \tau\left(z, n_{z}\right.$, $\left.2^{-i} \varepsilon\right)$. Therefore, Proposition 2.1 and Lemma 4.2 of [4] give in this case

$$
\begin{aligned}
|S(\zeta, z)| & \gtrsim-\operatorname{Re} S(\zeta, z)+|\operatorname{Im} S(\zeta, z)| \\
& \gtrsim-\operatorname{Re} \mu+3|\operatorname{Im} \mu|-|2 K \operatorname{Re} \mu \operatorname{Im} \mu|-c \sum_{j=2}^{m} \sum_{\alpha+\beta=j}\left|a_{\alpha \beta}(z, v)\right||\lambda|^{j} \\
& \gtrsim|\mu|-c k_{0}^{2} \sum_{j=2}^{m} \sum_{\alpha+\beta=j} \mid a_{\alpha \beta}(z, v) \tau^{j}\left(z, v, 2^{-i} \varepsilon\right) \\
& \gtrsim k_{1} \tau\left(z, n_{z}, 2^{-i} \varepsilon\right)-c k_{0}^{2} 2^{-i} \varepsilon \cong 2^{-i} \varepsilon .
\end{aligned}
$$

This finishes the proof of i). In order to show ii), we use that weget from Proposition 3.1 of [4] the following implications: there are uniform constants such that

$$
\begin{aligned}
\zeta \notin \frac{1}{2} P_{2^{-i} \varepsilon}(z) & \Rightarrow \zeta \notin P_{2^{-i} c \varepsilon}(z) \\
& \Rightarrow d(\zeta, z) \geq K c 2^{-i} \varepsilon \\
& \Rightarrow d(z, \zeta) \geq K K^{\prime} c 2^{-i} \varepsilon \\
& \Rightarrow z \notin P_{t}(\zeta) \forall t \leq K^{\prime \prime} K K^{\prime} c 2^{-i} \varepsilon \\
& \Rightarrow z \notin P_{K_{0} 2^{-i} \varepsilon}(\zeta) \\
& \Rightarrow z \notin K_{1} P_{2^{-i} \varepsilon}(\zeta) .
\end{aligned}
$$


On the other hand, there exists a constant $k_{1}>0$, such that for any point of the form $z^{\prime}=\mu^{\prime} n_{\zeta}+\lambda^{\prime} v^{\prime}$, the following two inequalities together

$$
\left|\mu^{\prime}\right| \leq k_{1} \tau\left(\zeta, n_{\zeta}, 2^{-i} \varepsilon\right) \text { and }\left|\lambda^{\prime}\right| \leq k_{1} \tau\left(\zeta, v^{\prime}, 2^{-i} \varepsilon\right)
$$

imply

$$
z^{\prime} \in K_{1} P_{2^{-i} \varepsilon}(\zeta)
$$

Hence, we have for a pair $(z, \zeta), z=\mu n_{\zeta}+\lambda v$, chosen as in ii) of the lemma necessarily

$$
|\mu| \geq k_{1} \tau\left(\zeta, n_{\zeta}, 2^{-i} \varepsilon\right) \text { or }|\lambda| \geq k_{1} \tau\left(\zeta, v, 2^{-i} \varepsilon\right) \text {. }
$$

Let us again choose any $k_{0}<k_{1}$ and suppose that $|\lambda| \geq k_{0} \tau\left(\zeta, v, 2^{-i} \varepsilon\right)$. Then we get from Proposition 2.1

$$
\begin{aligned}
\mid \rho(\zeta) & +S(z, \zeta) \mid \\
& \gtrsim-\rho(\zeta)-\operatorname{Re} S(z, \zeta) \\
& \gtrsim \frac{|\operatorname{Re} \mu|}{2}+\frac{K}{2}(\operatorname{Im} \mu)^{2}+c \sum_{j=2}^{m} \sum_{\alpha+\beta=j}\left|a_{\alpha \beta}(\zeta, v)\right||\lambda|^{j} .
\end{aligned}
$$

From (3.14) together with Proposition 3.1 of [4] we get

$$
|\rho(\zeta)+S(z, \zeta)| \gtrsim 2^{-i} \varepsilon
$$

We still have to consider the case $|\lambda| \leq k_{0} \tau\left(\zeta, v, 2^{-i} \varepsilon\right)$. Hence, necessarily, $|\mu| \geq k_{1} \tau\left(\zeta, n_{\zeta}, 2^{-i} \varepsilon\right)$. Therefore, we can estimate

$$
\begin{aligned}
|\rho(\zeta)+S(z, \zeta)| & \gtrsim-\rho(\zeta)-\operatorname{Re} S(z, \zeta)+|\operatorname{Im} S(z, \zeta)| \\
& \gtrsim \frac{|\operatorname{Re} \mu|}{2}+3|\operatorname{Im} \mu|-|2 K \operatorname{Re} \mu \operatorname{Im} \mu| \\
& -c \sum_{j=2}^{m} \sum_{\alpha+\beta=j}\left|a_{\alpha \beta}(\zeta, v)\right||\lambda|^{j} \\
& \gtrsim|\operatorname{Re} \mu|+|\operatorname{Im} \mu|-c k_{0}^{2} 2^{-i} \varepsilon \\
& \gtrsim k_{1} 2^{-i} \varepsilon-c k_{0}^{2} 2^{-i} \varepsilon \\
& \gtrsim 2^{-i} \varepsilon .
\end{aligned}
$$

This finishes the proof of the lemma.

With the aid off the last two lemmas we now can easily prove Lemma 3.1: 
Proof of Lamma 3.1. Since the family $\left\{P_{\varepsilon}^{i}(z)\right\}_{i=0}^{\infty}$ is a covering of $P_{\varepsilon}(z)$ we get

$$
\begin{aligned}
& \int_{D \cap P_{\varepsilon}(z)}|K(\zeta, z)| d V(\zeta) \\
\leq & \sum_{i=0}^{\infty}\left(\int_{D \cap P_{\varepsilon}^{i}(z)} \frac{\left|W_{1}(\zeta, z)\right|}{|S(\zeta, z)||\rho(\zeta)+S(z, \zeta)|^{n}} d V(\zeta)\right. \\
& \left.+\int_{D \cap P_{\varepsilon}^{i}(z)} \frac{\left|W_{2}(\zeta, z)\right|}{|S(\zeta, z)||\rho(\zeta)+S(z, \zeta)|^{n}} d V(\zeta)\right)
\end{aligned}
$$

Together with Lemma 3.3, parts i) and ii) this gives

$$
\begin{aligned}
& \int_{D \cap P_{\varepsilon}(z)}|K(\zeta, z)| d V(\zeta) \\
\leq & \sum_{i=0}^{\infty}\left(\frac{1}{\left(2^{-i} \varepsilon\right)^{n+1}} \int_{D \cap C_{1} P_{2-i \varepsilon}(z)}\left|W_{1}(\zeta, z)\right| d V(\zeta)\right. \\
& \left.+\frac{1}{\left(2^{-i} \varepsilon\right)^{n+1}} \int_{D \cap C_{1} P_{2}-i_{\varepsilon}(z)}\left|W_{2}(\zeta, z)\right| d V(\zeta)\right) .
\end{aligned}
$$

According to Lemma 3.2 applied to $\zeta \in C_{1} P_{2^{-i} \varepsilon}(z) \subset P_{c 2^{-i} \varepsilon}(z)$ we can continue this estimate as follows

$$
\begin{aligned}
\int_{D \cap P_{\varepsilon}(z)}|K(\zeta, z)| d V(\zeta) & \lesssim \sum_{i=0}^{\infty} \frac{1}{\left(2^{-i} \varepsilon\right)^{n+1}}\left(c 2^{-i} \varepsilon\right)^{1 / m+n+1} \\
& \lesssim \sum_{i=0}^{\infty} \varepsilon^{1 / m} 2^{-i / m} \cong \varepsilon^{1 / m}
\end{aligned}
$$

This proves Lemma 3.1.

\section{$\S 4$. Proof of Theorem 1.2}

Comparing what we have done so-far with [2], we can observe, that our Lemma 3.1 says, that the equation (24) from [2] still holds for all smooth convex domains of finite type. Furthermore, our Lemma 3.2 is the generalization of Lemma 4.3 of [2]. However, the rest of the proof of Theorem 1.2 is for us now very easy. The reader just has to convince himself, that the complete subsection 4.4 of [2] is only based on there equation (24) and there Lemma 4.3. Together with our Lemmas 3.1 and 3.2 it carries over literally to our more general situation. We do not repeat it here. 


\section{REFERENCES}

[1] B. Berndtsson and M. Andersson, Henkin-Ramirez formulas with weight factors, Ann. Inst. Fourier, 32 (1982), 91-110.

[2] J. Bruna, P. Charpentier and Y. Dupain, Zero varieties for the Nevanlinna class in convex domains of finite type in $\mathbb{C}^{n}$, Ann. Math. 147 (1998), 391-415.

[3] A. Cumenge, Zero sets of functions in the Nevanlinna and Nevanlinna-Drjbachian classes in convex domains of finite type, To appear in Pacific J. Math.

[4] K. Diederich, B. Fischer and J. E. Fornæss, Hölder estimates on convex domains of finite type, Math. Z. 232 (1999), 43-61.

[5] K. Diederich and J. E. Fornæss, Support functions for convex domains of finite type, To appear in Math. Z., 1999.

[6] K. Diederich and J. E. Fornæss and J. Wiegerinck, Sharp hölder estimates for $\bar{\partial}$ on ellipsoids, Manuscripta math. 56 (1986), 399-417.

[7] G. M. Henkin, H. Lewy's equation and analysis on pseudoconvex manifolds I, Uspehi Mat. Nauk 32 (1977), no. 3, 57-118.

[8] J. McNeal, Estimates on the Bergman kernel of convex domains, Adv. Math. 109 (1994), 108-139.

[9] H. Skoda, Valeurs au bord pour les solutions de l'opérateur $\bar{\partial}$, et caractérisation des zéros des fontions de la classe de Nevanlinna, Bull. Soc. Math. France 104 (1976), 225-299.

Klas Diederich

Mathematik

Universität Wuppertal

Gausstr. 20

D-42097 Wuppertal

Germany

klas@uni-wuppertal.de

Emmanuel Mazzilli

Département de Mathématiques

CNRS URA D751

Université Lille 1

F-59655 Villeneuve d'Ascq

France

emmanuel.mazzilli@agat.univ-lille1.fr 